\title{
Plasma-chemical synthesis of $\mathrm{Fe}_{3} \mathrm{O}_{4}$ nanoparticles for doping of high-temperature superconductors
}

\author{
A.V. Uschakov ${ }^{a, b}$, I.V. Karpov ${ }^{a, b}$, A.A. Lepeshev ${ }^{a, b} *$ \\ ${ }^{a}$ Krasnoyarsk Scientific Center of the Siberian Branch of the Russian Academy of Science, \\ 660036, Krasnoyarsk, Russia \\ ${ }^{b}$ Siberian Federal University, 660041, Krasnoyarsk, Russia \\ ${ }^{c}$ Kirensky Institute of Physics, Russian Academy of Sciences, 660036, Krasnoyarsk, Russia \\ * Corresponding author. \\ E-mail address: sfu-unesco@mail.ru (A.A. Lepeshev)
}

\begin{abstract}
Ferrite nanoparticles $\left(\mathrm{Fe}_{3} \mathrm{O}_{4}\right)$ have been produced by the direct low pressure plasma-chemical synthesis. X-ray diffraction (XRD), high-resolution transmission electron microscopy (HRTEM), vibration magnetometry (VSM), Mössbauer spectroscopy (NGR) were used for measurements, showing that the produced nanoparticles have an average size of $9.4 \mathrm{~nm}$, a crystalline phase of magnetite, possess a property of superparamagnetism at room temperature and have a blocking temperature of $89 \mathrm{~K}$. The peculiarities of nanoparticles behavior in the magnetic field, related to a large specific surface area, are discussed.
\end{abstract}

Keywords: Ferrite nanoparticles, low pressure plasma-chemical synthesis, X-ray diffraction, transmission electron microscopy, vibration magnetometry, Mössbauer spectroscopy.

Introduction. Magnetic nanoparticles are recently widely investigated due to their unusual properties that make them attractive both from the point of basic characteristics as well as for potential applications in various technological fields, such as means for magnetic information recording, sensors based on the effect of the giant magnetoresistance and ferro-fluids. Use of magnetic nanoparticles as minor additives to improve the properties of high temperature superconductors are of particular interest [1-4]. The works [5-7] show that the magnetite nanoparticles are effective pinning centers and contribute to a significant increase in the critical current $J_{c}$ in granular high-temperature superconductors. Therefore, simple and cost-effective reproducible methods for production of nanoparticles of various materials are extremely important for the development of nanotechnology [8-13]. The most widely spread chemical methods $[14,15]$ are inefficient and quite complex to be implemented, besides the nanoparticles, being produced, are contaminated by products of chemical reactions. Plasma-chemical methods for production of magnetic nanoparticles are the most appropriate [16, 17]. The presence in the plasma of supersaturated vapors of the initial compounds and the high rate of their hardening, 
under certain conditions, results in nanoparticles formation. Changing the energy of the particles in the condensation process allows producing various structures of a specific material from amorphous up to crystalline ones. Meanwhile, the size and the shape of the crystals vary depending on the energy. This work is aimed at finding the features of the structural-phase state and magnetic properties of nanoparticles based on ferrites, produced in the low pressure plasma arc discharge.

Materials and methods. The phase composition of the samples was investigated using the Advance D8 X-ray diffractometer in monochromatic $\mathrm{CuK} \alpha$ radiation. The scanning was carried out at room temperature in the angular range $30-120 \mathrm{deg}$ in $2 \theta$ with a step of $0.04 \mathrm{deg}$.

The microstructure of the produced nanoparticles was analyzed by using JEOL JEM-2100 transmission electron microscope (at an accelerating voltage of $200 \mathrm{keV}$ ). The samples for the electron microscopy study were prepared as follows: the powder was placed in isopropyl alcohol and then it was dispersed in an ultrasonic bath for 2 minutes, and a solution drop was then placed on a carbon film substrate, located on an electron-microscopic supporting grid. The carbon film substrate was $10-15 \mathrm{~nm}$ thick.

The magnetic properties of nanopowder were analyzed using vibrating magnetometry by means of a universal strong field measuring system Cryogenic at room temperature in the magnetic fields of $0 \pm 20,000$ Oe. The range of the field stabilization was 1 Oe. The saturation magnetization $M s$ was analyzed by extrapolating the function $M\left(H^{-1}\right)$ at $1 / H \rightarrow 0$ as described in [18].

Mössbauer studies of the alloys were carried out at $78 \mathrm{~K}$ and $294 \mathrm{~K}$ by means of nuclear gamma resonance spectrometer of electrodynamic type in a constant acceleration mode with ${ }^{57} \mathrm{Co} / \mathrm{Rh}$ source. In order to calibrate the isomer shifts and speed scale, $\alpha$-Fe foil was used at 294 $\mathrm{K}$.

The experimental setup along with the dependence of the powder properties on the conditions of sputtering was considered in detail in [19]. The arc evaporator had the following characteristics: an arc current was $100 \mathrm{~A}$ and arc stabilization on the cathode surface was carried out by means of an electrostatic screen. A cathode, made of iron $(99,99)$, was used as a sputtering cathode. In order to begin plasma chemical reactions, the chamber was preliminary pumped out to a pressure of $1 \mathrm{mPa}$ and then it was filled by a gas mixture containing $30 \% \mathrm{O}_{2}+70 \%$ Ar. Plasma-chemical synthesis was carried out under the gas mixture pressure of 70 $\mathrm{Pa}$. The cathode was heated to $500 \mathrm{~K}$ before evaporation. The powders were produced under these pressures for $10 \mathrm{~min}$. The produced powder was condensed and collected on a cooled substrate made of stainless steel. The substrate temperature remained constant at $300 \mathrm{~K}$. Below describes the operating parameters of the reactor during the synthesis. 
Operating parameters of a vacuum plasma-chemical reactor for ferrite nanoparticles synthesis:

- Basic pressure in chamber $10^{-3} \mathrm{~Pa}$

- Arc discharge current $100 \mathrm{~A}$

- Voltage $70 \mathrm{~V}$

- Power $10 \mathrm{~kW}$

- Plasma-forming gas (argon) supply $70 \mathrm{~Pa}$

- Reaction gas (oxygen) supply 30 vol. $\% \mathrm{O}_{2}$

Results and discussion. Figure 1 shows images of high-resolution transmission electron microscopy, the particle distribution diagrams and the density functions of the size distribution of nanoparticles being produced.

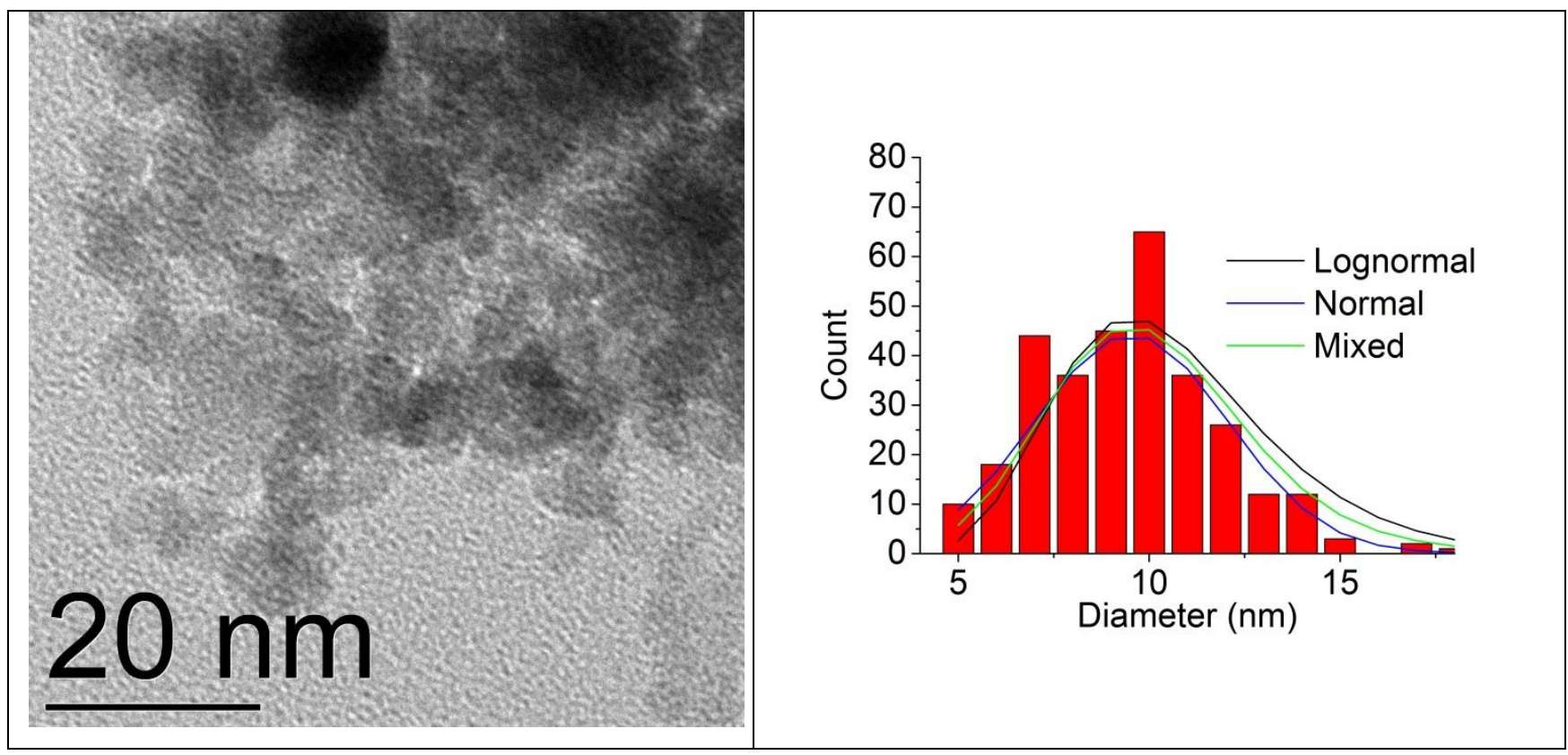

Figure 1. TEM images, histograms and probability density functions of nanopowders produced under the pressure $70 \mathrm{~Pa}$ of the gas mixture $\left(30\right.$ vol. $\left.\% \mathrm{O}_{2}+70 \% \mathrm{Ar}\right)$

It is easy to conclude from the above results, that the produced powder represents strongly agglomerated particles of spherical shape. The particle size ranges from 5 to $20 \mathrm{~nm}$. The combination of normal and log-normal size distribution are characterized for these particles. This suggests two competing mechanisms of particle condensation from steam-plasma phase: cluster condensation and steam condensation. The given mixed density function represents the sum of density functions of lognormal and normal distribution with adjustable parameters. As can be seen from the figure above, this function describes the particle size distribution much better. The 
average particle size was $9.4 \mathrm{~nm}$, the mean mass particle size was $10.2 \mathrm{~nm}$ and the standard deviation was 1.18 .

Nanopowders are characterized by a high surface energy, which is balanced out by a significant aggregation of powders, which causes a significant reduction in the specific surface. It is well known [20],that particle size distribution function is directly related to the process of nanoparticles formation. The distribution function can differ significantly for the different methods of nanopowders production. There are two main mechanisms for nanoparticles formation: diffusive and coagulative.

Since nanoparticle synthesis in plasmachemical reactor is always accompanied by chemical processes, so the size distribution function of nanoparticles gets more complicated because of the products of these reactions. The morphology of the particles also gets more complicated and particles of different chemical composition are formed. Besides, mutual diffusion of nanoparticles in the condensed phase occurs. Under the sufficiently high temperature, multiple processes can occur simultaneously, forming the strong coupling between nanoparticles. The experimental data are described by a normal distribution within prevailing of nanoparticles layered growth due to the adsorption of atoms and diffusion processes during the atomic mass transfer at the interface [21].

The X-ray diffraction pattern for the values of $2 \theta$ in the range from $25^{\circ}$ to $65^{\circ}$ is shown in Fig. 2. The reflexes can be determined within the diffraction pattern corresponding to the structure of maghemite $\gamma-\mathrm{Fe}_{2} \mathrm{O}_{3}$ (PDF \# 391346, space group P 2 13 ) with the lattice parameter $a$ $=8.339$, hematite $\alpha-\mathrm{Fe}_{2} \mathrm{O}_{3}$ (PDF \# 330664, space group R $\overline{3} \mathrm{c}$ ) with the lattice parameter $a=5.01$, $c=13.68$, magnetite $\mathrm{Fe}_{3} \mathrm{O}_{4}$ (PDF \# 190629, space group $\mathrm{F} \mathrm{d} 3 \mathrm{~m}$ ) with the lattice parameter $a=$ 8.388. Other crystalline structures have not been found. The presence of amorphous phase is due to the peculiarity of the plasma-chemical synthesis, in which the saturation of the nanoparticles with oxygen occurs in a thermodynamically non-equilibrium conditions, under violation of stoichiometry, high residual stresses and defects in the crystal structure. 


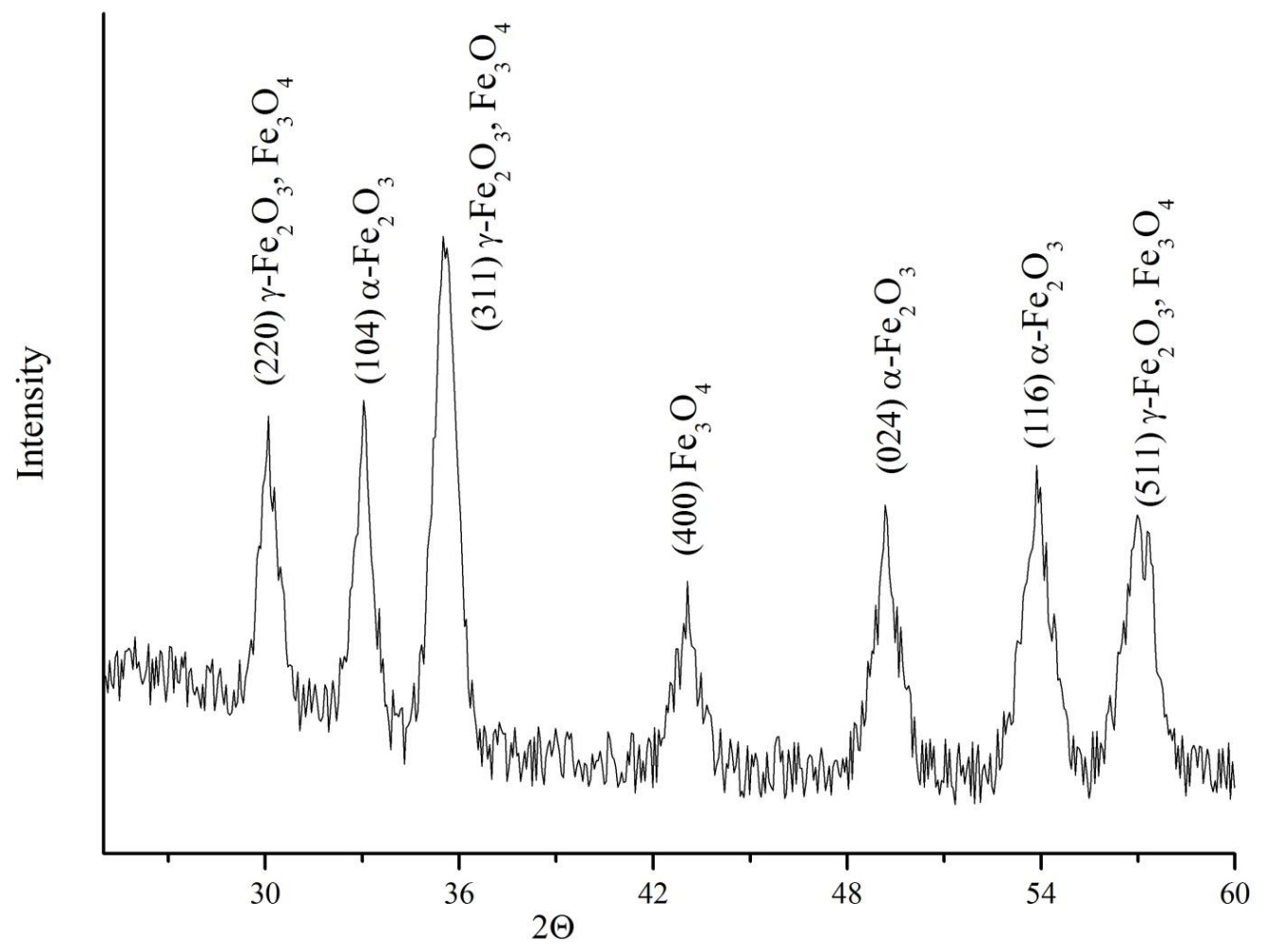

Figure 2. XRD pattern of the produced nanopowder

The lattice parameter is slightly smaller than the standard value, that is most probably caused by the peculiarity of condensation under the low pressure. Deformation of the particles can occur as a result of influence of the capillary forces. Static displacements, caused by non-uniform nature of deformation in small particles, are mostly contributed by the mean-square displacement. The abrupt nature of crystallization process causes incompleteness of forming the crystal lattice and implies increased concentration of non-equilibrium vacancies. The influence of the additional surface pressure as a result of free energy excess for small particles leads to nonuniform structural deformation in the volume of a particle. The microstructural characteristics and the elementary cell parameters were determined by using X-ray full-profile analysis by means of the Rietveld method [22]. The particularities of X-ray peaks showed the presence of two fractions in the sample, significantly different in crystallite size. This conclusion was based on the fact that the diffraction maxima have anomalously sharp peaks under the large integrated width. This complex shape could be explained only by a superposition of two peaks, significantly different in width. Therefore, for a full-profile adjustment two fractions with different crystallite size and different composition percentage were introduced to the model. Full-profile adjustment of microstructural parameters revealed that the majority of the sample ( $98 \%$ ) is the fraction with an average crystallite size of $9 \mathrm{~nm}$, which agrees well with the data obtained from microscopic studies. In order to clarify the dispersion of the producing powder, a 
specific surface of recently deposited nanoparticles was studied by the BET method. The studies were carried out based on the specific retention volume, determined from the gas chromatograms of thermal desorption. The mean diameter of the nanoparticles according to the equation $\mathrm{D}_{\mathrm{av}}=$ $6 / \rho \mathrm{S}_{\mathrm{sp}}$, was determined equal to $2 \mathrm{~nm}$. Thus, the produced nanoparticles have a highly developed surface, which is characteristic for conditions of the condensation from supersaturated vaporplasma flows.

Precise interpretation of X-ray is difficult because of the broad overlapping peaks and the possible presence of maghemite and magnetite phases, with reflexes being coincided. For a more precise solution of the problem of powders phase composition, it is preferable to conduct an additional Mössbauer spectroscopy study. It is well known [23] that, Mössbauer spectra of iron nuclei of $\mathrm{Fe}_{3} \mathrm{O}_{4}$ and $\gamma-\mathrm{Fe}_{2} \mathrm{O}_{3}$ phases are rather different primarily due to the fact that the first oxide includes divalent and trivalent iron ions, and the maghemite includes only trivalent ones. Fig. 3, $a$ shows the experimental spectrum of the produced powder at room temperature.

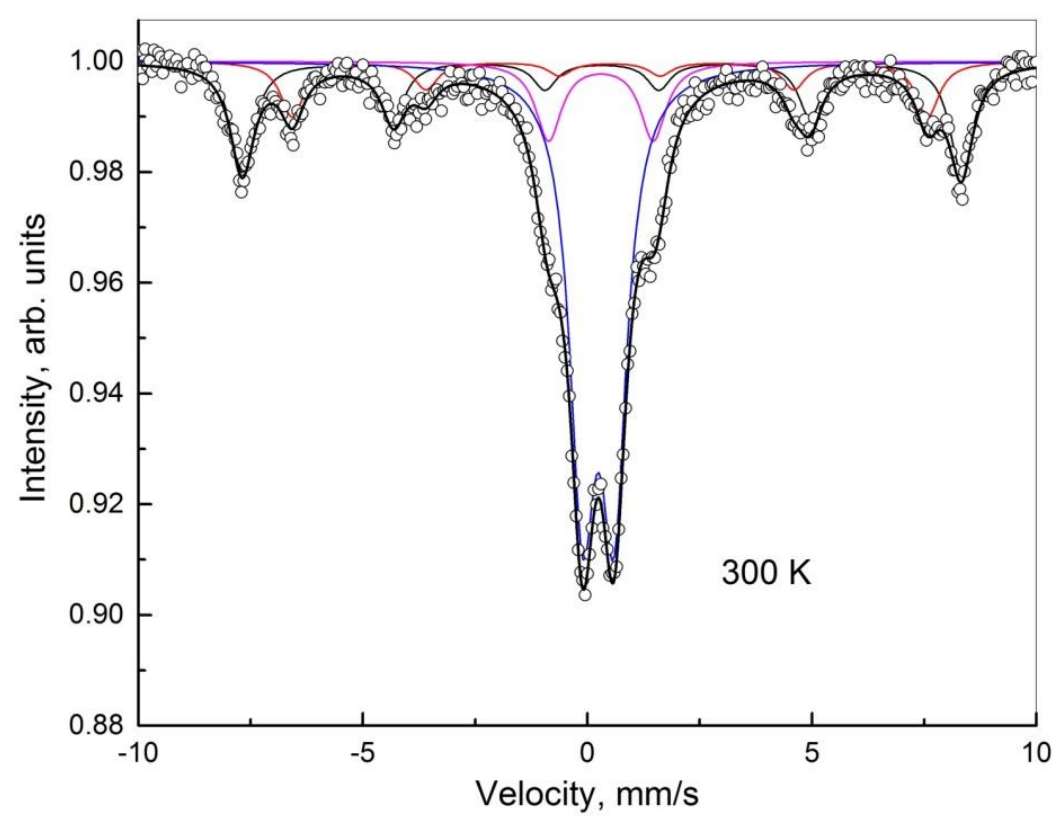

a) 


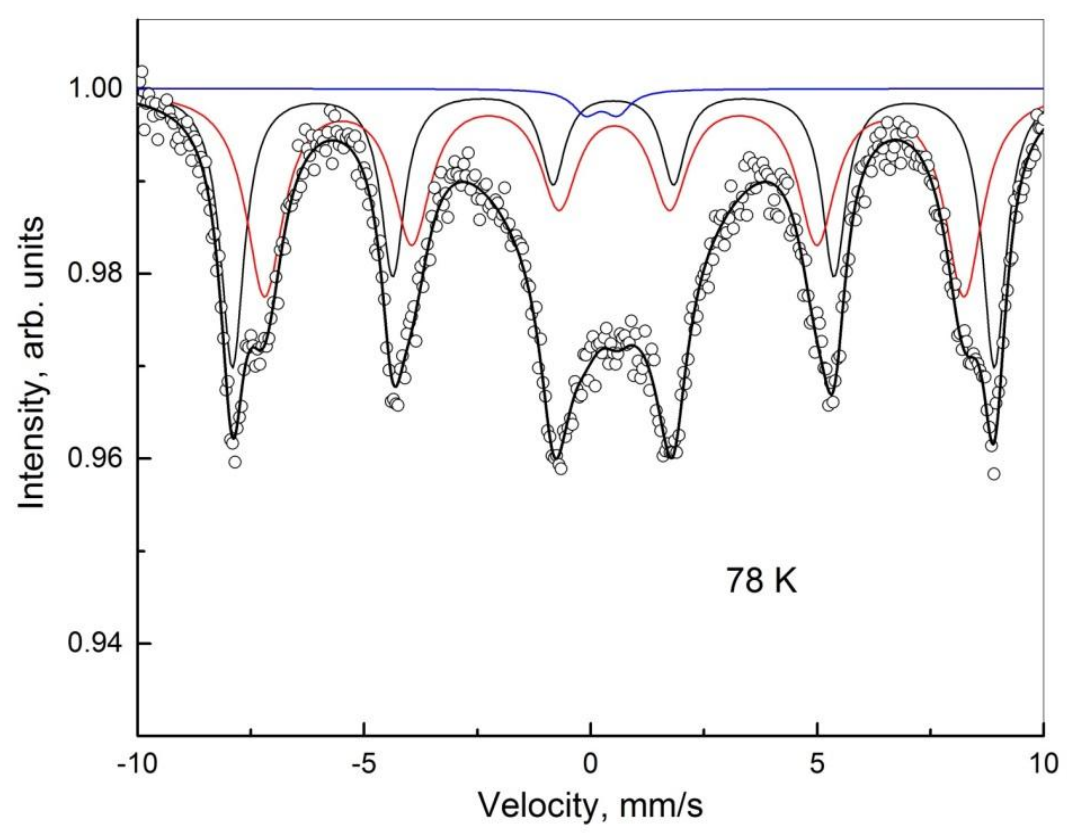

b)

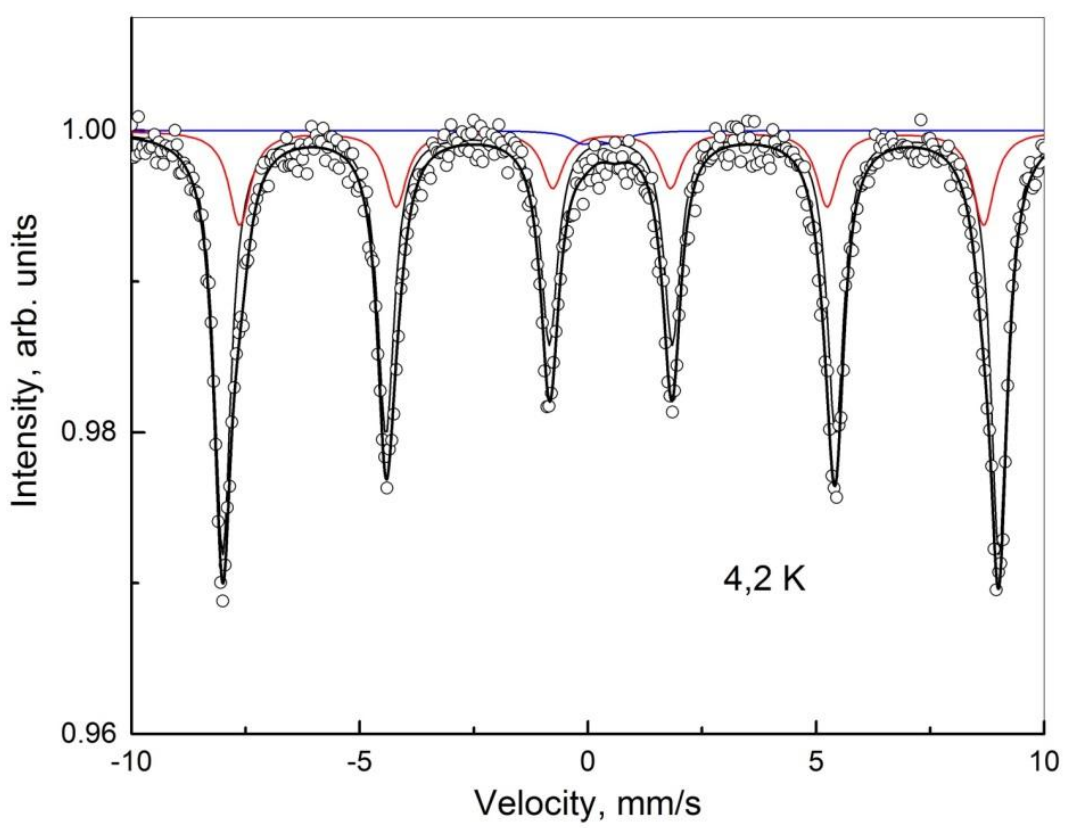

c)

Figure 3. Mössbauer spectra of ${ }^{57} \mathrm{Fe}$ nuclei in the structure of the nanoparticles at different temperatures

The analysis revealed that the spectra of the produced nanopowders, containing dispersed particles with an average size of about $9 \mathrm{~nm}$, were sufficiently symmetric relative to its "center", and were characterized by large width of the lines. The spectrum processing was carried out using the simultaneous model decoding of the quadrupole doublet and the distribution analysis of hyperfine magnetic field (Table 2). Mössbauer spectrum represents a superposition of doublets: the intense doublet located in the central part, the low intensity doublet and two low- 
intensity sextets, which is characteristic of superparamagnetic particles. The intensive doublet with the average parameters $\langle\mathrm{IS}\rangle=0.24 \mathrm{~mm} / \mathrm{s}$ and $\langle\mathrm{QS}\rangle=0.71 \mathrm{~mm} / \mathrm{s}$ corresponds to the positions of ferric iron atoms in a tetrahedral surrounding by oxygen atoms. While the doublet of low intensity with the average parameters $\langle\mathrm{IS}\rangle=0.27 \mathrm{~mm} / \mathrm{s}$ and $\langle\mathrm{QS}\rangle=2.33 \mathrm{~mm} / \mathrm{s}$ corresponds to the position of ferrous iron atoms. This is likely to occur in the case of nonequilibrium distribution of cations, which is characterized by two states of iron ions: magnetically ordered and magnetically disordered, leading to both strengthening and weakening the value of the exchange interaction.

The appearance of the quadrupole doublet is most probably related to the formation of a new phase on a surface of nanocomposite particles; however, the contribution of superparamagnetic relaxation should also be viewed.

Mössbauer spectra measurements were performed at a temperature of $5 \mathrm{~K}$ for a more precise identification of the iron atoms phase (Fig. 3,c). Processing the spectra was performed using a model consisting of two Zeeman sextets and a quadrupole doublet. Zeeman sextet with an average Mössbauer pilot shift $\langle\mathrm{IS}>=0.48$ corresponds to the position of ferric iron atoms in a tetrahedral environment of oxygen, and a value $\langle$ IS $>=0.55$ corresponds to the position of atoms of divalent and trivalent iron in an octahedral environment of oxygen atoms [24]. It should be noted, that the relative intensity of the lines of the first and the second sextets in $\mathrm{Fe}_{3} \mathrm{O}_{4}$ should be close to 1:2 in accordance with the distribution of atoms in the lattice positions. However, in the investigated powders the relative intensity of these lines was much more different from the intensity mentioned above. One of the reasons is most probably a nonstoichiometry of the phase composition, specifically represented by the formula $\mathrm{Fe}_{3-\mathrm{x}} \mathrm{O}_{4}$, where $x$ are the number of vacancies [21]. The presence of these structural defects probably explains the observed difference in the relative intensities of certain lines on the experimental and simulated (calculated) powders diffraction patterns.

Mössbauer measurements were carried out at a temperature of $78 \mathrm{~K}$ (Fig. 3, $b$ ). The spectra represent the Zeeman sextets with the widened lines characteristic of superparamagnetic particles. The more is the temperature of spectrum measurement the broader are the lines of magnetic hyperfine structure being observed. This is explained by an increase in the frequency of superpamagnetic relaxation due to the increased energy of thermal fluctuations of the magnetic moment. The analysis of the spectra showed that the effective magnetic field on the nucleus is decreased, which is characterized by a shift of the hyperfine structure components to the center of the spectrum. A specific shift of the maximum location in the hyperfine magnetic field distribution at the nucleus area was observed. 
Table 1. Parameters of hyperfine interactions obtained within mathematical processing of experimental Mössbauer spectra of samples

\begin{tabular}{|c|c|c|c|c|}
\hline \multirow{5}{*}{$300 \mathrm{~K}$} & $\mathrm{IS}, \mathrm{mm} / \mathrm{s}$ & $\mathrm{QS}, \mathrm{mm} / \mathrm{s}$ & $H_{\text {eff }}, \mathrm{T}$ & $S, \%$ \\
\cline { 2 - 5 } & $0.326 \pm 0.002$ & $0.016 \pm 0.001$ & $49.843 \pm 0.053$ & 30.5 \\
\cline { 2 - 5 } & $0.521 \pm 0.001$ & $0.022 \pm 0.006$ & $44.014 \pm 0.074$ & 23.8 \\
\cline { 2 - 5 } & $0.241 \pm 0.001$ & $0.710 \pm 0.013$ & - & 11.5 \\
\cline { 2 - 5 } & $0.715 \pm 0.004$ & $2.334 \pm 0.027$ & - & 34.2 \\
\hline \multirow{3}{*}{$78 \mathrm{~K}$} & $0.506 \pm 0.014$ & $0.116 \pm 0.003$ & $52.376 \pm 0.036$ & 57.1 \\
\cline { 2 - 5 } & $0.522 \pm 0.008$ & $0.005 \pm 0.001$ & $48.061 \pm 0.048$ & 42.9 \\
\hline \multirow{2}{*}{$4.2 \mathrm{~K}$} & $0.505 \pm 0.012$ & $0.002 \pm 0.001$ & $52.901 \pm 0.025$ & 68.4 \\
\cline { 2 - 5 } & $0.523 \pm 0.007$ & $0.003 \pm 0.002$ & $50.711 \pm 0.023$ & 31.6 \\
\hline
\end{tabular}

The values of a hyperfine parameter $\alpha$, equal to the ratio of the energy of magneto-crystalline anisotropy to the energy of thermal fluctuations of magnetic moment $K_{e f f} V / k_{\mathrm{b}}$, were obtained from the low-temperature studies. The evaluation of particle size was carried out based on these studies. The nanocomposite particle sizes $d=10.5 \pm 0.1 \mathrm{~nm}$ were estimated within the model of spherical particles with a diameter $d$ and by using the model for the effective anisotropy constant [25].

Zero-field-cooled (ZFC) magnetization and field-cooled (FC) magnetization were measured at applied field 100 Oe (Figure 4).

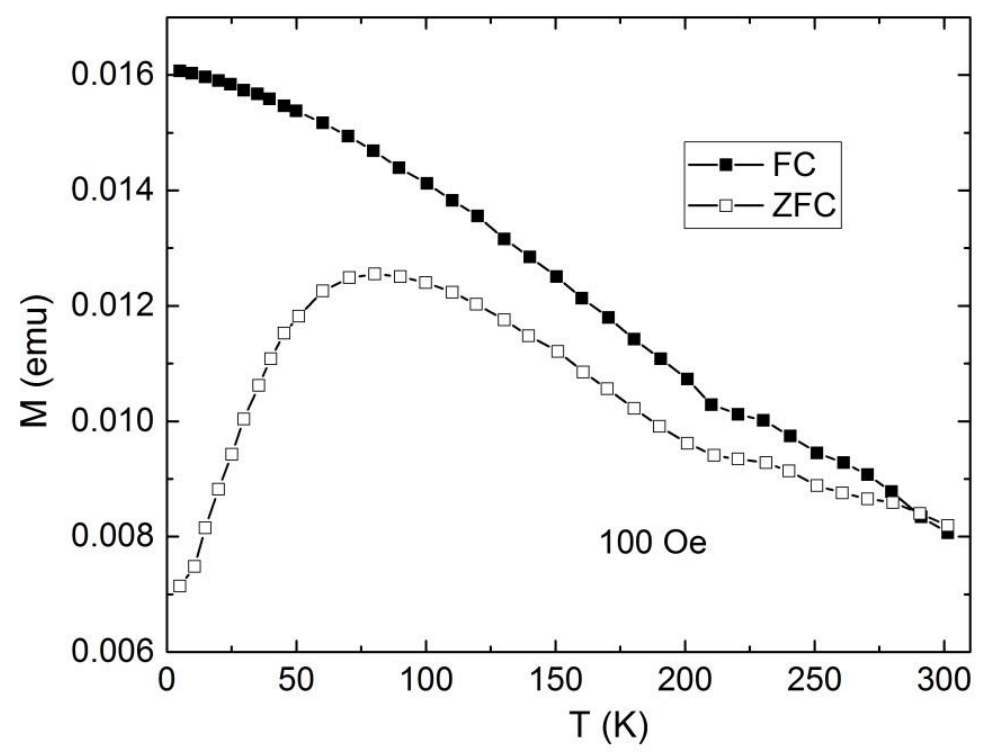

Figure 4. ZFC and FC magnetization as function of temperature were measured at applied field 100 Oe. 
The behavior of ZFC curve is characteristic of mictomagnetism of superparamagnetic particles with blocking temperature $T_{b}=86 \mathrm{~K}$. A special feature of the curves is the lack of bifurcation temperature in the studied range, that implies the fact of blocking the magnetic moments at room temperature. For superparamagnetic systems a bifurcation temperature of ZFC and FC magnetization curves satisfies the inequality $T_{\mathrm{bf}} \geq \mathrm{T}_{\mathrm{b}}$. Parameter $\mathrm{T}_{\mathrm{bf}}$ can be interpreted as the starting point of nanoparticles magnetization formation for the states analogous to a spinglass and superparamagnetic phenomenon.

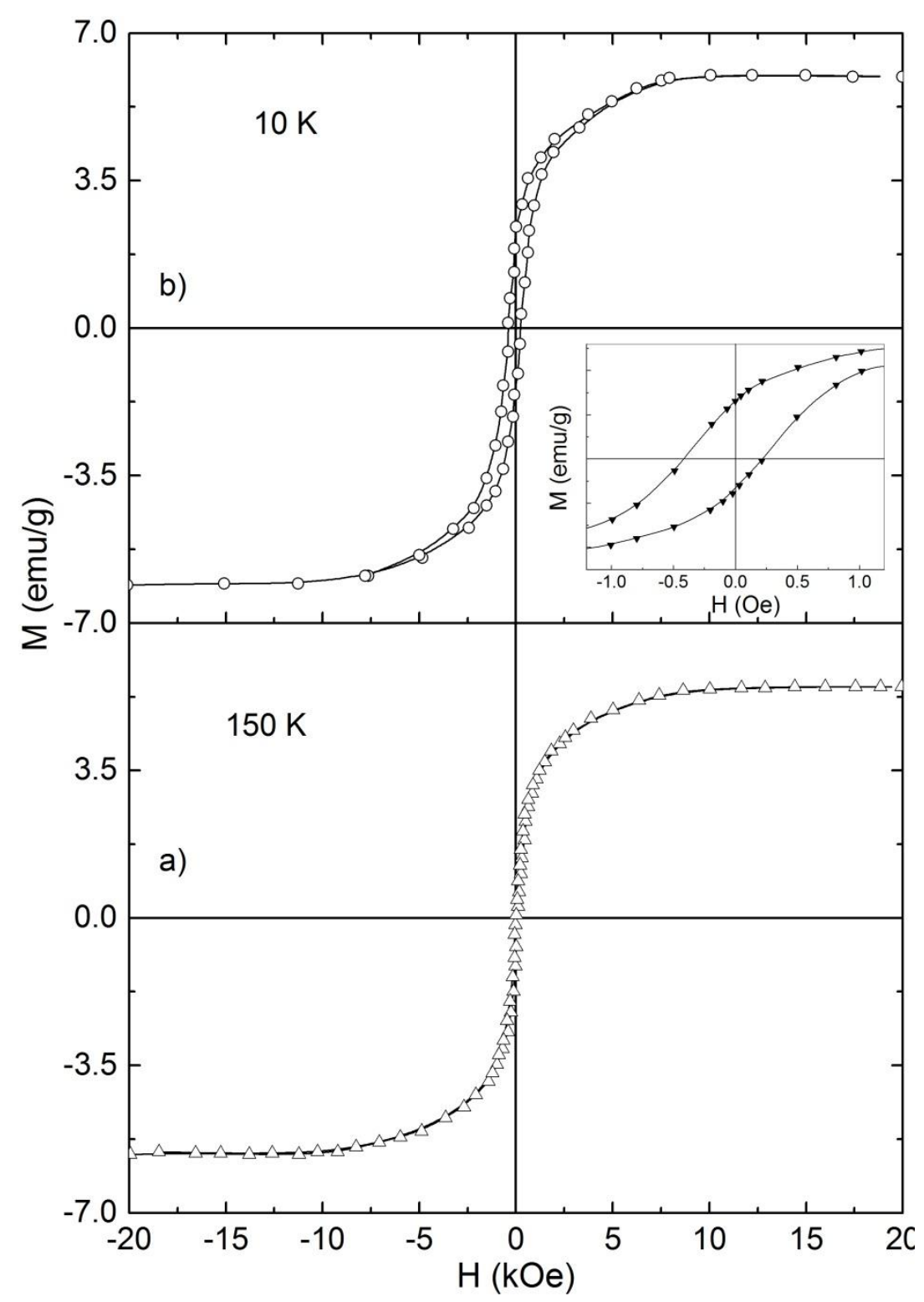

Figure 5. Hysteresis curve at temperatures of 10 and $150 \mathrm{~K}$

For superparamagnetic systems $T_{b}{ }^{1 / 2}$ linearly depends on the field $H$, while parameter $T_{b}$ for spin glasses systems decreases proportionally to $H^{2 / 3}$. For electric arc single-domain systems with the properties of superparamagnetism or spin glass, $T_{b}$ is expected to behave in a similar 
way [26]. It should be noted that the peak on the temperature ZFC-dependence of magnetization is narrow enough, indicating small distribution of particles by sizes and absence of a magnetic interaction between the nanoparticles in the ensemble. Figure 5 shows the dependence of the magnetization on the applied magnetic field at temperatures of 10 and $150 \mathrm{~K}$.

It is quite clear, that at $10 \mathrm{~K}$ the magnetic hysteresis curve is seen, which is characteristic for ferromagnetic materials possessing a coercive force of $H_{c}=360$ Oe and a ratio of residual magnetization to saturation magnetization $\left(R=M_{\mathrm{R}} / M_{\mathrm{S}}\right)$ of approximately 0.26 . The increase in the measurement temperature leads to a decrease of the coercive force to zero, while the sample exhibits superparamagnetic properties. The relatively low value of $R$ in accordance with [27] means that the system should be antiferromagnetic and interaction between the nanoparticles occurs. Furthermore, the produced nanoparticles have less value of $M_{\mathrm{s}}$ than that of the particles with large volume. Authors of [28] found a linear relationship between parameter $M_{s}$ and sizes of the nanoparticles; they explained it by the highly developed surface. According to the studies of the specific surface, there is a clusters layer of $\sim 2 \mathrm{~nm}$ in size on each nanoparticle, which partially prevents dipole-dipole interaction between particles, despite their strong agglomeration.

Conclusion. The peculiarities of plasma-chemical synthesis of ferrite nanoparticles in the plasma of low pressure, related to the mixed nature of condensation, have been found. Transmission electron microscopy, X-ray diffraction and specific surface measurements showed that the particles have an average size of $9.4 \mathrm{~nm}$ and a highly developed surface with a thickness of $2 \mathrm{~nm}$. Mössbauer studies at temperatures of $4.2 \mathrm{~K}, 78 \mathrm{~K}$ and $300 \mathrm{~K}$ confirmed phase composition and particle size distribution of the produced nanoparticles and showed that the particles remained to possess superparamagnetic properties in a wide range of temperatures. Vibration magnetometry studies revealed a transition from ferromagnetic to superparamagnetic state of nanoparticles and allowed to find the blocking temperature of $89 \mathrm{~K}$ and the coercive force $H_{c}=360 \mathrm{Oe}$. The investigation of the $\mathrm{ZFC}$ and $\mathrm{FC}$ magnetization as function of temperature were measured at applied field 100 Oe showed the absence of bifurcation point in the analyzed range. The produced nanomaterial has a potential to be used for high-temperature superconductors synthesis.

\section{Acknowledgments}

The work was performed with a support of the grant of the Russian Science Foundation (Project No. 16-19-10054) 


\section{References}

1. A. Snezhko, T. Prozorov, R. Prozorov, Phys. Rev. B 71 (2005) 024527. doi: 10.1103/PhysRevB.71.024527.

2. X. Dong, P. An, J. Zhang, H. Zhang, Y. Li, H. Liu, X. Ge, Q. Li, J of Superconductivity and Novel Magnetism 27 (2014) 693. doi: 10.1007/s10948-013-2343-9.

3. A.V. Ushakov, I.V. Karpov, A.A. Lepeshev, M.I. Petorv, L.Yu. Fedorov, JETP Letters 99 (2014) 99. doi: 10.1134/S002136401402009X.

4. A.V. Ushakov, I.V. Karpov, A.A. Lepeshev, M.I. Petrov, J. Appl. Phys. 118 (2015) 023907. http://dx.doi.org/10.1063/1.4926549.

5. E. Babić, N. Novosel, Physica C: Superconductivity $493 \quad$ (2013) 119. doi:10.1016/j.physc.2013.03.043.

6. I.M. Obaidat, B.A. Albiss, H. Ghamlouche, S.M. Obeidat, T.G. Qarqaz, M. Gharaibeh, A. Obeidat, M.K. Hasan, J. Nanoeng. Nanomanuf. 1 (2011) 84. doi: http://dx.doi.org/10.1166/jnan.2011.1006.

7. S.N. Abd-Ghani, R. Abd-Shukor, W. Kong, Advanced Materials Research 501 (2012) 309.

8. I.V. Karpov, A.V. Ushakov, A.A. Lepeshev, S.M. Zharkov, Vacuum 128 (2016) 123. doi: 10.1016/j.vacuum.2016.03.025.

9. A.A. Lepeshev, I.V. Karpov, A.V. Ushakov, G.E. Nagibin, J of Alloys and Compounds 663 (2016) 631. doi:10.1016/j.jallcom.2015.12.168.

10. A.A. Lepeshev, I.V. Karpov, A.V. Ushakov, L.Yu. Fedorov, A.A. Shaikhadinov, International Journal of Nanoscience 15 (2016) 1550027.

11. L.Yu. Fedorov, I.V. Karpov, A.V. Ushakov, A.A. Lepeshev, Inorganic Materials. 51 2015 25. DOI: 10.1134/S0020168515010057.

12. A.V. Ushakov, I.V. Karpov, A.A. Lepeshev, Technical Physics 86 (2016) 260. doi: $10.1134 / \mathrm{S} 1063784216020262$.

13. A.V. Ushakov, I.V. Karpov, A.A. Lepeshev, Physics of the Solid State 57 (2015) 2320. doi: 10.1134/S1063783415110359.

14. M.M. Lin, D.K. Kim, A.J. El Haj, J. Dobson, IEEE transactions on Nanobioscience 7 (2008) 298. http://dx.doi.org/10.1109/TNB.2008.2011864.

15. J.B. Mamani, L.F. Gamarra, G.E. de Souza Brito Mat. Res. 17 (2014) 3. http://dx.doi.org/10.1590/S1516-14392014005000050.

16. A.V. Ushakov, I.V. Karpov, A.A. Lepeshev, M.I. Petrov, L.Yu. Fedorov, Physics of the Solid State. 57 (2015) 919. doi: 10.1134/S1063783415050303. 
17. A.V. Ushakov, I.V. Karpov, A.A. Lepeshev, L.Yu. Fedorov, A.A. Shaikhadinov, Technical Physics. 86 (2016) 103. doi: 10.1134/S1063784216010230.

18. S.R. Ahmed, S.B. Ogale, G.C. Papaefthymiou, R. Ramesh, P. Kofinas, Appl. Phys. Lett. 80 (2002) 1616. http://dx.doi.org/10.1063/1.1456258.

19. I.V. Karpov, A.V. Ushakov, L.Yu. Fedorov, A.A. Lepeshev, Technical Physics 84 (2014) 559. doi: 10.1134/S1063784214040148.

20. A.N. Kolmogorov, USSR Report Academy of Sciences, 31 (1941) 99.

21. D.I. Ryzhonkov. Ultra-dispersed medium. Production of nanopowders by chemical dispersion and their properties Moscow, 2007.

22. H.M. Rietveld, J. Appl. Crystallogr. 2 (1969) 65.

23. I.P. Suzdalev. Dinamic effects in gamma-resonance spectroscopy, Moscow, 1979.

24. G.F. Goya, T.S. Berquo, F.C. Fonseca, M.P. Morales, J. Appl. Phys. 94 (2003). 3520. http://dx.doi.org/10.1063/1.1599959.

25. F. Bodker, S. Morup, S. Linderoth, Phys. review letters 72 (1994) 282. http://dx.doi.org/10.1103/PhysRevLett.72.282.

26. R.K. Zheng, H. Gu, B. Xu, X.X. Zhang, J. Phys.: Condens. Matter. 18 (2006) 5905. http://dx.doi.org/10.1088/0953-8984/18/26/010.

27. E.C. Stoner, E.P. Wohlfarth, Philosophical Transactions of the Royal Society A. 240 (1948) 599. doi: 10.1098/rsta.1948.0007.

28. L.C. Varanda, P.Jr. Jafelicci, K. O’Grady, T. González-Carreño, M.P. Morales, T. Muñoz, C.J. Serna, J. Appl. Phys. 92 (2002) 2079. http://dx.doi.org/10.1063/1.1496124. 